\title{
El Bitcoin: una revisión de las ventajas y desventajas de las transacciones comerciales con dinero virtual
}

\author{
Antonio Rodas Portillo ${ }^{1}$ \\ antoniorodasportillo758@gmail.com \\ Sergio Ariel Núñez Gill ${ }^{2}$ \\ snunezgill@gmail.com \\ Docente tutora metodológico: \\ Msc. María Dolores Muñoz \\ Facultad de Ciencias Contables, Administrativas y Económicas \\ UNIVERSIDAD NACIONAL DE PILAR
}

\section{RESUMEN}

El bitcoin es considerado una nueva forma de utilizar dinero para los intercambios de bienes, servicios e inversiones, desde plataformas digitales. La investigación tiene como objetivo identificar las ventajas y desventajas de transacciones comerciales con bitcoin, utilizando una metodología documental para traer a la actualidad el estado del arte del tema en cuestión desde las perspectivas de investigadores. Para el desarrollo de la investigación se realizó una búsqueda en Google académico con las palabras clave: bitcoin, dinero virtual, ventajas y desventajas, donde se encontraron como resultado, aproximadamente 984 estudios sobre el tema. Se seleccionaron 46 artículos para definir las ventajas y desventajas del Bitcoin, como sistema de intercambio monetario a nivel digital. El análisis documental realizado permite identificar como principales ventajas: su globalidad, transacciones realizadas en tiempo real, altos estándares de seguridad y riesgos, no sufre fluctuaciones de inflación, le da al usuario la libertad de pagos, así como de enviar y recibir cualquier cantidad de dinero de forma instantánea, con tasas muy bajas en comparación con los productos financieros y las principales desventajas son: las más visibles son la volatilidad, otra desventaja es la garantía de aceptación, pues aunque cada vez hay más establecimientos que los aceptan todavía son una minoría, otra desventaja es el hecho de que no cuenta con respaldo de activos físicos o emisores legales.

Palabra clave: bitcoin; moneda virtual; ventajas; desventajas.

\footnotetext{
${ }^{1}$ Tesinando de la carrera de Contaduría Pública Nacional

${ }^{2}$ Tesinando de la carrera de Contaduría Pública Nacional
} 


\title{
Bitcoin: a review of the advantages and disadvantages of virtual money trading transactions
}

\begin{abstract}
Bitcoin is considered a new way of using money for the exchange of goods, services and investments, from digital platforms. The research aims to identify the advantages and disadvantages of commercial transactions with bitcoin, using a documentary methodology to bring to the present the state of the art of the subject in question from the perspectives of researchers. For the development of the research, an academic Google search was carried out with the keywords: bitcoin, virtual money, advantages and disadvantages, where approximately 984 investigations in this regard were found as a result. 46 articles were selected to define the advantages and disadvantages of Bitcoin, as a digital currency exchange system. The documentary analysis carried out allows us to identify the main advantages: its globality, transactions carried out in real time, high standards of security and risks, does not suffer from inflation fluctuations, gives the user the freedom of payments, as well as to send and receive any amount of money instantly, with very low rates compared to financial products and the main disadvantages are: the most visible are volatility, another disadvantage is the guarantee of acceptance, because although more and more establishments accept them, they are still a minority, another disadvantage is the fact that they do not have the backing of physical assets or legal issuers.
\end{abstract}

Keyword: bitcoin; virtual currency; advantages; disadvantages.

Artículo recibido: 02 noviembre. 2021 Aceptado para publicación: 28 noviembre 2021 Correspondencia: antoniorodasportillo758@gmail.com Conflictos de Interés: Ninguna que declarar 


\section{INTRODUCCIÓN}

El Bitcoin es un fenómeno global, pues según el informe del Índice Global de Adopción de Criptomonedas, tiene aceptación y uso en 154 países a nivel mundial. El informe destaca que Ucrania es el país que encabeza este ranking, seguido por Rusia, Venezuela y China. Se trata de países en desarrollo, pero, no obstante, carecen de un sistema bancario-comercial afianzado (Callejo, 2020).

Los países desarrollados se encuentran introduciendo el comercio con criptomonedas, por el contrario, la "fiebre" del Bitcoin en los mercados en desarrollo, como los de América Latina, aún están intentando: pues según los datos de bitValor (bolsa de Brasil), en 2016 las ventas bursátiles en las monedas de Bitcoin crecieron un 322\%, al igual que en la bolsa mexicana (Naúmenko \& Fakhrutdínova, 2019).

En Paraguay, estudios relacionados al Bitcoin demuestran que los encuestados conocen el Bitcoin; desean conocer más sobre el mismo, creen que es seguro utilizar el Bitcoin como medio de pago, lo consideran rentables y prácticos para la sociedad, e inclusive estarían de acuerdo con el uso del Bitcoin como medio de pago. (Ibarra Muñoz, 2019).

Desde el punto de vista del planteamiento metodológico, se advierte que la presente investigación es de tipo exploratorio con base documental; lo que se ha hecho es elaborar un estado del arte en el que se analiza el fenómeno del Bitcoin, sus ventajas y desventajas, desde varias perspectivas de autores, quienes investigaron al respecto.

La ruta metodológica que se ha seguido ha comprendido básicamente tres momentos: exploración, focalización y profundización.

La importancia del estudio, se debe a que en los últimos años el Bitcoin ha crecido en el mercado digital, amenazando drásticamente la manera tradicional que siguen las transacciones de dinero, bienes y servicios (Agila, 2019). Es posible que la "moneda virtual" represente la emergencia de un nuevo paradigma o la forma de comercializar lo que podría poner "en jaque:" al dinero tangible que conocemos actualmente. Esta situación provoca una preocupación en los economistas, financieros, administradores de empresas y contadores, pues el bitcoin está amenazando las profesiones que comúnmente se dedican a la administración y gestión del dinero (Farías \& Espitia, 2018). Dando paso a una era donde, quienes estén a la vanguardia de los cambios financieros e implicancia digital, sean lo que puedan responder a lo que promete ser la futuro moneda (Orrala \& Chompol, 2017). 
Ante esta perspectiva, y con la intención de conocer este nuevo fenómeno financiero, el presente estudio tiene como objetivo identificar las ventajas y desventajas de transacciones comerciales con bitcoin.

\section{MARCO REFERENCIAL}

\subsection{Una aproximación al concepto de Bitcoin}

El bitcoin es un tema bastante analizado en la actualidad debido a su importante crecimiento en los últimos años, y porque se trata de un sistema de dinero digital que no cuenta con políticas de estado a nivel nacional o internacional, y que tampoco los respaldan las corporaciones dedicadas a las transacciones financieras. Pero antes de avanzar, es importante saber cómo definen los estudiosos a esta nueva moneda de cambio. Sánchez (2021), define al Bitcoin como la moneda virtual o criptomoneda que sirve como medio de compra y venta de bienes o servicios entre las personas, sin tener en cuenta la nacionalidad o los límites geográficos a nivel mundial. Al respecto, González (2018), lo ve como un activo que se ha instituido incuestionablemente como una atrayente oportunidad y una alternativa válida en el mundo digital para ahorrar e invertir.

El Bitcoin en palabras llanas se puede definir como la nueva moneda digital encriptada y descentralizada impulsado por sus usuarios sin una autoridad o intermediarios, que se utiliza como factor de cambio, mediante el uso de la web (Medina, Zarate y López, 2020). Por su parte, Armijos (2015), define al Bitcoin, al igual que Sánchez (2021), como una moneda virtual e intangible, que no se puede palpar, pero que en ciertas plataformas digitales sirve como medio de pago, que, si bien puede aumentar o disminuir dentro de una cuenta digital, esta no puede ser monetizada.

Así coincide en su definición Canales, (2020), pues también se refiere al Bitcoin como moneda virtual, este autor señala que fue creada por una persona cuya identidad no ha sido revelada y que bajo el alias de "Satoshi Nakamoto" maneja esta nueva moneda virtual, sin que exista un mecanismo central que la gobierne, sino que el andamio de usuarios que poseen o utilizan estas monedas, son suficientes para la gestión y el mantenimiento del producto. Lo que despierta una gran desconfianza entre los conocedores del mundo financiero, pues para animarse a usar esta forma de pago, moneda o transacción, es necesario tener confianza, pues como señala autores anteriores a este párrafo, no cuenta con un marco regulatorio, tampoco con apoyo de entidades gubernamentales o bancarias. 
Para Canales (2020), al no contar con un mecanismo de control, se habla de una situación extremadamente volátil, por lo que este autor no es recomienda usarla sin conocimientos previos, de cómo funciona y menos, si se desea invertir, por ende, se debe conocer el riesgo en que se incurre.

El Bitcoin es considerada como la moneda digital con potencial capaz homologar los problemas de cambios de moneda, es decir, este sistema digital permite pasar una moneda digital dos veces (Reyes y Joancomartí,2016). Esto sirve principalmente para realizar transacciones económicas, tales como comprar, vender e invertir sin importar el rubro o cambio de la economía al que se pretende introducir, es considerada verdad mente una innovación que ayuda a minimizar los costes financieros de las transacciones y elimina la forma tradicional de gestionar el dinero, dejando de lado los productos bancarios (Montoya Ramírez, 2020).

Para Martín (2019), lo inusual de esto, y lo que más llama la atención, es que a pesar de ser criptomonedas, es decir están encriptadas; son monedas que pueden ser como una moneda tradicional, proporcionándoles un alto grado de seguridad por el hecho de que es imposible su falsificación. Para Carrera-López, Sánchez-Lunavictoria, y Loza-Torres (2020), el Bitcoin es la criptomoneda considerada por los visionarios tecnológicos, como la más importante del mundo digital, pudiendo acentuarse en varios países del mundo, donde empresas comerciales han aceptado a esta moneda digital, como una forma más de pago, debido al auge de su movimiento en el ámbito digital.

Rodríguez Gómez (2020), considera que lo que hace atractivo a este nuevo sistema de dinero, es el carácter limitado y la escasez absoluta de Bitcoin convierten a la criptomoneda en un activo peculiar, con cualidades muy interesantes desde el punto de vista inversor relacionadas con su ratio rentabilidad-riesgo y su efecto diversificador. Adicionalmente, el carácter disruptivo de su tecnología subyacente -la blockchain- y la atención que suscita entre grandes empresas tecnológicas y organismos gubernamentales parece empujar más si cabe el interés por la criptomoneda.

\subsection{Aspecto jurídico del Bitcoin.}

En el aspecto jurídico, el sistema digital de intercambio de moneda de bitcoin no cuenta con una ley de circulación oficial, pero, como señala Cajamarca Andrade y Curipoma Narváez (2020), son tratadas como bienes muebles debido a que las operaciones de compra y venta de criptomonedas no se encuentran prohibidas, estas transacciones se 
pueden realizar en las plataformas correctamente reguladas como lo son: Libertex, eToro, ExpertOption, entre otras

Teniendo en cuenta la Ley de la oferta y la demanda, el Bitcoin sigue esta línea comercial y su valor se establece a través de un algoritmo algebraico (dentro de una plataforma digital) que calcula la cantidad de movimientos y transacciones en tiempo real (Reyes Asanza y Rosero Banchon, 2018). Esto lo convierte en una moneda fungible: lo que significa, que tiene similitud de función con la moneda material, ya que el Bitcoin se consume o se gasta con el uso, pero, al contrario de la moneda tradicional, una transacción con esta criptomoneda, la tecnología no te permite su uso nuevamente. No obstante, sigue siendo el Bitcoin una moneda incorporal, por no estar representado en el mundo físico como algo tangible y visible, pero, concordando con Canales Gutiérrez (2018), sigue siendo una moneda descentralizada de curso voluntario que aumenta sus seguidores en el mundo virtual.

Entre los Aspectos tributarios de las transacciones en criptomonedas, al ser criptodivisas, una de sus características es que no cuentan con curso legal en jurisdicción alguna, es decir que no tienen soporte por ninguna autoridad monetaria o banca central y no se encuentran soportadas por activos físicos, razón por la cual se pueden entender como medios de intercambio digitales que cuentan con un valor asignado por el mercado y permiten realizar la compra y venta de bienes y servicios (Sánchez, Ruiz, Osorio, López, Arredondo, Carazo y López (2019).

Inaudiblemente, los estudios e informes analizados en el presente artículo, demuestran que el Bitcoin se potencia como la herramienta monetaria más competitiva contra el sistema financiero tradicional, y que, a pesar de su entorno especulativo, está provocando, como señala Agila Suárez (2019), una revolución en los ámbitos comerciales, turísticos, políticos y socioeconómicos

\section{3. ¿Cómo funciona el Bitcoin?}

El Bitcoin se trata de una moneda digital, que se puede negociar a través de Internet, y por ello se considera como la primera moneda digital descentralizada. En principio, este mecanismo de pago se puede usar para cualquier tipo de transacción y se puede aplicar a cualquier activo que se pueda representar en forma digital (Compains, García y Campo, 2021). Para Rosales, Maledo y García (2017), por su arquitectura el Bitcoin puede operar libremente y tiene el potencial para ser implementado globalmente, es una alternativa para 
hacer pagos y enviar dinero por todo el mundo de forma libre, sin horarios, sin bancos, con completo control y con comisiones muy bajas por sus características digitales, lo que lo vuelve atractivo para los negocios y divisas internaciones.

Un análisis de la estabilidad paramétrica de series de tiempo para el periodo 2009-2018 desarrollado por Licona y Sosa (2019) demuestra que el bitcoin es un medio de cambio digital que por medio de tecnología criptográfica otorga validez y confiabilidad a sus transacciones, pero sigue siendo un bien sumamente riesgoso, tiene el valor que el inversionista esté dispuesto a pagar por él. Como se ha mencionado, a menudo tiene más que ver con psicología humana que con cálculos económicos o financieros. Los altos niveles de interés del público pueden exagerar la acción en el precio; los informes periodísticos de los precios crecientes del bitcoin atraen inversionistas especulativos y desinformados.

Si bien, el Bitcoin es una moneda electrónica descentralizada que se crea en 2008, en un análisis teórico-práctico de la moneda virtual Bitcoin y sus implicaciones económicas permitieron a Sánchez Leonsegui (2018) explicar que su funcionamiento se basa en la tecnología de la cadena de bloques, que no es más que un libro de contabilidad compartido entre todos los usuarios de la red.

Según estudios realizados por Gracia (2018), el bitcoin es una moneda virtual que permite a dos partes hacer una transacción directamente entre ellos sin la necesidad de un tercero de confianza. Se trata de dinero digital diseñado para funcionar en un mundo sin intermediarios. Se sustenta en la tecnología Blockchain, que posee el potencial para revolucionar la economía mundial. Así mismo coincide Juanpere (2019), quien describe a esta moneda digital, con capacidad de realizar transacciones en línea, punto a punto, de forma descentralizada y sin la participación de ninguna institución financiera. Desde el punto de vista tecnológico, su funcionamiento se basa en el uso de algoritmos matemáticos, el cifrado mediante doble clave asimétrica y la tecnología blockchain, donde cada usuario dispone de dos claves, una pública y otra privada, mediante las cuales se asegura la seguridad de las transacciones sin que intervengan terceros y permite su despliegue a través de los bloques de datos de la cadena que son inalterables.

Según Vázquez-Gutiérrez (2018), los Bitcoins han atraído demasiado la atención en los últimos años y ha generado que el precio de estas monedas remonte de manera 
impresionante e incontrolable. Muchos investigadores piensan que estas monedas son el futuro de la economía por sus múltiples ventajas que tienen el utilizarlas.

Una publicación sobre la Tributación del bitcoin, que hace un estudio de comparación entre Colombia y Estados Unidos, explica que este tipo de moneda es una especie del género denominado moneda virtual, y se mantendrá así, toda vez que su proceso de creación y validación como medio de cambio, no se sujeta a la potestad de una autoridad gubernamental central, que verifique su autenticidad y validez dentro de un país o comunidad (Lozano y Forero, 2017).

Independientemente a las críticas, sustentos y evidencias relacionadas a las ventajas y desventajas del Bitcoin, actualmente es considerado por sus usuarios como un buen medio de cambio, aunque con un alto nivel de riesgo en la función de depósito de valor y una unidad de medida bastante pobre (Muñoz Esteban, 2017).

\section{MATERIALES Y MÉTODOS}

El presente trabajo corresponde a un estudio teórico descriptivo de carácter exploratorio, de tipo documental, dado que el procedimiento implica el rastreo, organización, sistematización y análisis de Artículos Científicos realizados entre los años 2015-2021 publicados y accesibles en la web, cuyo contenidos abarcan ventajas y desventajas que ofrece la moneda digital.

Las unidades de análisis fueron los siguientes Artículos seleccionados por relación y aporte al tema de estudio:

\begin{tabular}{|c|c|}
\hline & \\
\hline $\begin{array}{l}\text { Sánchez, S. G. O. (2021). } \\
\text { Educación financiera basada en } \\
\text { el bitcoin y la inclusión en planes } \\
\text { de estudio }\end{array}$ & $\begin{array}{l}\text { mericana para la Investigación y el } \\
\text { 11(22). } \\
\text { (2021). Educación financiera basada } \\
\text { clusión en planes de estudio. RIDE } \\
\text { a para la Investigación y el Desarrollo }\end{array}$ \\
\hline 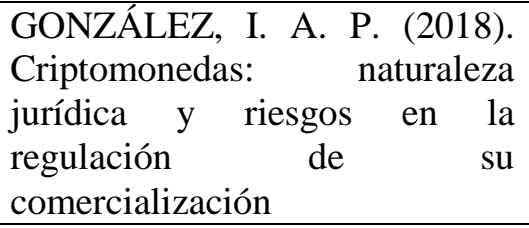 & $\begin{array}{l}\text { Revista de derecho del mercado de valores, }(22), 5 \text {. } \\
\text { Link: GONZÁLEZ, I. A. P. (2018). Criptomonedas: } \\
\text { naturaleza jurídica y riesgos en la regulación de su } \\
\text { comercialización. Revista de derecho del mercado de } \\
\text { valores, (22), 5. }\end{array}$ \\
\hline $\begin{array}{l}\text {. Las monedas } \\
\text { transacciones } \\
\text { análisis sobre el } \\
\text { temas de pagos }\end{array}$ & $\begin{array}{l}\text { (2015). Las monedas virtuales en las } \\
\text { erciales. Un análisis sobre el uso de } \\
\text { pagos. Research Gate. }\end{array}$ \\
\hline $\begin{array}{l}\text { LAGUENS ARTIGAS, L. U. C. } \\
\text { A. S. \& SON LAS MONEDAS } \\
\text { VIRTUALES EL DINERO DEL }\end{array}$ & 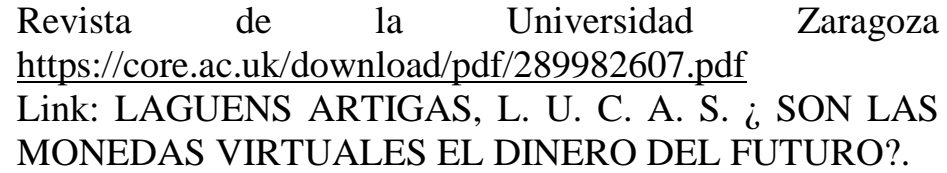 \\
\hline
\end{tabular}




\begin{tabular}{|c|c|}
\hline $\begin{array}{ll}\text { Canales, M. P. (2020). } & \text { El } \\
\text { BITCOIN: Comprendiendo } & \text { el } \\
\text { Mercado. } & \end{array}$ & $\begin{array}{l}\text { oomía y sociedad, 1(1), 9-24. } \\
\text { 020). El BITCOIN: Comprendiendo } \\
\text { economía y sociedad, 1(1), 9-24. }\end{array}$ \\
\hline $\begin{array}{l}\text { Reyes, M. F. M., \& Joancomartí, } \\
\text { J. H. Análisis Y Comparación De } \\
\text { Monedas Criptográficas Basadas } \\
\text { En La Tecnología Blockchain. }\end{array}$ & $\begin{array}{l}\text { Biblioteca de la Universidad Autónoma de Barcelona. Tesis } \\
\text { Magistral } \\
\text { Link: Reyes, M. F. M., \& Joancomartí, J. H. Análisis Y } \\
\text { Comparación De Monedas Criptográficas Basadas En La } \\
\text { Tecnología Blockchain. }\end{array}$ \\
\hline $\begin{array}{lcr}\begin{array}{l}\text { Montoya } \\
(2020) \text {. El }\end{array} \text { bitcoin } & \text { una } \\
\text { innovación } & & \\
\text { financiera (Bachelor's tesis) }\end{array}$ & $\begin{array}{l}\text { Escuela de Economía, Administración y Negocios. Facultad } \\
\text { de Negocios Internacionales). } \\
\text { Link: Montoya Ramírez, J. D. (2020). El bitcoin una } \\
\text { innovación financiera (Bachelor's thesis, Escuela de } \\
\text { Economía, Administración y Negocios. Facultad de } \\
\text { Negocios Internacionales). }\end{array}$ \\
\hline $\begin{array}{l}\text { Medina, M. A. C., Zarate, C. V., } \\
\text { \& López, L. M. (2020). La } \\
\text { necesidad de la regulación } \\
\text { contable, legal y fiscal del bitcoin } \\
\text { en México, un tema de } \\
\text { actualidad. }\end{array}$ & $\begin{array}{l}\text { Revista Horizontes de la Contaduría en las Ciencias } \\
\text { Sociales, (13). } \\
\text { Link: Medina, M. A. C., Zarate, C. V., \& López, L. M. } \\
\text { (2020). La necesidad de la regulación contable, legal y fiscal } \\
\text { del bitcoin en México, un tema de actualidad. Horizontes de } \\
\text { la Contaduría en las Ciencias Sociales, (13). }\end{array}$ \\
\hline $\begin{array}{l}\text { Carrera-López, J. S., Sánchez- } \\
\text { Lunavictoria, J. C., \& Loza- } \\
\text { Torres, A. G. (2020). El uso de } \\
\text { las criptomonedas como nueva } \\
\text { forma de pago en la economía } \\
\text { mundial. }\end{array}$ & $\begin{array}{l}\text { Revista Científica FIPCAEC (Fomento de la investigación y } \\
\text { publicación en Ciencias Administrativas, Económicas y } \\
\text { Contables). ISSN: 2588-090X. Polo de Capacitación, } \\
\text { Investigación y Publicación (POCAIP), 5(5), 210-223. } \\
\text { Link: Carrera-López, J. S., Sánchez-Lunavictoria, J. C., \& } \\
\text { Loza-Torres, A. G. (2020). El uso de las criptomonedas } \\
\text { como nueva forma de pago en la economía mundial. Revista } \\
\text { Científica FIPCAEC (Fomento de la investigación y } \\
\text { publicación en Ciencias Administrativas, Económicas y } \\
\text { Contables). ISSN: 2588-090X. Polo de Capacitación, } \\
\text { Investigación y Publicación (POCAIP), 5(5), 210-223. }\end{array}$ \\
\hline $\begin{array}{l}\text { Agila Suárez, R. J. (2019). El } \\
\text { Bitcoin: Crecimiento y } \\
\text { desarrollo de la criptomoneda y } \\
\text { su inclusión como alternativa de } \\
\text { pago y medio de intercambio en } \\
\text { la economía ecuatoriana, } \\
\text { período: } 2014-2018\end{array}$ & $\begin{array}{l}\text { (Bachelor's thesis, Universidad de Guayaquil. Facultad de } \\
\text { Ciencias Económicas). } \\
\text { Link: Agila Suárez, R. J. (2019). El Bitcoin: Crecimiento y } \\
\text { desarrollo de la criptomoneda y su inclusión como } \\
\text { alternativa de pago y medio de intercambio en la economía } \\
\text { ecuatoriana, período: 2014-2018 (Bachelor's thesis, } \\
\text { Universidad de Guayaquil. Facultad de Ciencias } \\
\text { Económicas). }\end{array}$ \\
\hline $\begin{array}{l}\text { Saldarriaga, V. P. (2019). } \\
\text { Lavado de activos mediante } \\
\text { criptomonedas en el Perú: } \\
\text { problemas y alternativas. }\end{array}$ & $\begin{array}{l}\text { Lex: Revista de la Facultad de Derecho y Ciencia Política de } \\
\text { la Universidad Alas Peruanas, 17(24), 161-178. } \\
\text { Link: Saldarriaga, V. P. (2019). Lavado de activos mediante } \\
\text { criptomonedas en el Perú: problemas y alternativas. Lex: } \\
\text { Revista de la Facultad de Derecho y Ciencia Política de la } \\
\text { Universidad Alas Peruanas, 17(24), 161-178. }\end{array}$ \\
\hline
\end{tabular}


Como criterios de búsqueda, se incluyeron los siguientes descriptores: "bitcoin”, "dinero virtual", "moneda virtual", "criptomoneda", "ventajas", "desventajas", "arquitectura" y "leyes". Estos descriptores fueron combinados de diversas formas al momento de la exploración con el objetivo de ampliar los criterios de búsqueda.

Al realizar la búsqueda de los documentos relacionadas al bitcoin, se halló aproximadamente 984 investigaciones al respecto. Teniendo en cuenta cada una de las bases de datos, se preseleccionaron 60 artículos, de los cuales se escogieron 46 (cuarenta y seis), de acuerdo con los criterios de inclusión y exclusión. No se tomaron en consideración para el análisis aquellos artículos que no hacían alusión a los núcleos temáticos y/o, en este caso el "Bitcoin" y a aquellos que no se encontraban en revistas indexadas.

Para la organización de los documentos, se creó una base de datos en Excel, con los siguientes campos (categorías de análisis): título del artículo, autor, año, revista, información de la revista, problema de investigación, objetivos, tipo de investigación, método, descripción y tamaño de la muestra, instrumentos utilizados, resultados y núcleo temático. Una vez organizada la información, se agruparon los documentos en tres: 1) Concepto de Bitcoin, 2) Aspecto jurídico del Bitcoin y 3) ¿Cómo funciona el bitcoin?, núcleos temáticos abordados en investigaciones, donde se describía a las ventajas y desventajas de la utilización del sistema de Bitcoin.

Posteriormente, se realizó el análisis de cada uno de los núcleos temáticos, identificando las ventajas y desventajas que exponían los autores, definiendo lo más relevante y describiendo los aspectos comunes y divergentes entre los documentos seleccionados, mediante un ejercicio de comparación constante. Finalmente se realizó un análisis global mediante el cual se identificaron las convergencias y divergencias del análisis de cada uno de los núcleos temáticos, se formularon ciertas hipótesis y conclusiones y se hicieron algunas recomendaciones.

\section{RESULTADOS Y DISCUSIÓN}

En este apartado se presentan la síntesis de los resultados y los aspectos más relevantes encontrados en los documentos, los cuales se explican y discuten para comprender las convergencias y divergencias de los autores y permiten presentar el estado del arte, respecto a las ventajas y desventajas del Bitcoin como moneda virtual para las transacciones comerciales. 


\subsection{Ventajas de las transacciones comerciales con Bitcoin}

Autores como Orrala \& Chompol (2017), Kim (2017), López, Restrepo, \& Ocampo (2020), Forero \& Sanabria (2017), Torres (2015), y Melguizo (2019) coinciden que, entre las ventajas principales del Bitcoin se encuentran su globalidad, en el sentido de que no pertenece a ningún estado o gobierno, pues se puede utilizar en todo el mundo, independientemente de las barreras geográficas y políticas; además, es ajeno al sistema fiduciario, puesto que, no se puede crear una deuda con él, ya que, su valor no depende de una intervención de un banco central; otra ventaja mencionada es que, las transacciones se realizan en tiempo real, pues puede estar realizada en menos de una hora, del mismo modo otra ventaja es que las plataformas que han aceptado el uso de Bitcoin garantizan cumplir con estándares de seguridad y riesgos y que los algoritmos matemáticos sustentan la confianza de este modelo.

Mientras que entre las ventajas encontradas por Lemos (2020), Triviño \& López (2020) Criollo \& Maldonado (2021), Agila (2019) y Farías \& Espitia (2018) se observa, la disminución de un $0,4 \%$ a 9,8\% en los costos de transacción como tipo de cambio con otras monedas reales, al igual que, la facilidad de acceso únicamente cibernético por lo que no se requiere de sistemas comerciales complicados y no podemos olvidar el ahorro en gastos y costos inherentes a las transacciones interbancarias como la gran cantidad de infraestructura, sistemas de negociación y salarios de empleados. También señala como ventajas la libertad de pagos, ya que, puede enviar y recibir cualquier cantidad de dinero de forma instantánea, en cualquier momento y desde cualquier lugar del mundo; las tasas son muy bajas, pues actualmente los pagos realizados con Bitcoin son procesados con tasas bajas o sin tasa alguna, existe menores riesgos para los comerciantes, en el sentido que, son seguras, irreversibles, y no contienen datos personales y privados de los clientes, al mismo tiempo que es neutral y transparente, ya que toda la información sobre el suministro de Bitcoin está disponible en la cadena de bloques para cualquiera que quiera verificarlo y usarlo. Igualmente, coinciden en que el hecho de que no haya intermediarios en las transacciones, supone una gran ventaja, pues no existe el pago por comisiones a ninguna entidad por el uso del dinero o por las transferencias realizadas, las transacciones se realizan en tiempo récord, el dinero no es embargable, y además no existe ningún costo de almacenamiento. Esto se debe a que el Bitcoin opera bajo el sistema peer to peer, el cual intercambia la información de manera directa, es decir, de cliente a servidor al mismo 
tiempo, lejos de la intervención del Estado o cualquier otro agente financiero que quiera fungir como intermediario, por lo que no es necesario el pago de comisiones a terceros. Otras de las, quizás más importantes ventajas según los autores, es que el Bitcoin se maneja bajo un sistema descentralizado, lo que permite la transparencia en los pagos, desde cualquier parte del mundo., lo que ha hace que el tiempo de ejecución de las operaciones sea más rápida ya que no se expone a congelación de fondos, y al ser un sistema de cifrado, es altamente seguro, lo que lo convierte en resistente a caídas de Red o ataques informáticos, presenta transparencia, pues las transacciones son públicas debido a que suceden en tiempo real, la ruta hacia dónde va cada bitcoin es públicamente visible, por lo que, los procesos se mantienen limpios y legales.

Los autores también coinciden en que el Bitcoin tiene una ventaja muy importante para los inversores, por su libre disponibilidad de intercambio con las divisas tradicionales o por otras divisas convencionales tales como el dólar, euro, yen, rublo, etc; tomando en consideración la tasa de cambio correspondiente, al mismo tiempo mencionó que es un sistema de pago de carácter internacional con rapidez, puesto que, la plataforma permite realizar transferencias a nivel internacional de manera más rápida y veloz, a diferencia del modelo tradicional que suelen tardar varios días.

Otra oportunidad mencionada por estos mismos autores, fue que no necesita requisitos previos, a diferencia del sistema financiero tradicional que solicita afiliación de cuentas y documentación para trámites en la red, el Bitcoin solo requiere de un dispositivo electrónico y la descarga de una App gratuita.

Por otro lado, Pérez (2018), Oña (2019) e Izquierdo (2018) coinciden que, entre sus ventajas destaca la integridad del sistema, la cual no se encuentra recargada sobre personas o instituciones, sino sobre los procedimientos matemáticos que han probado ser bastante eficientes y seguros, los cuales dentro su programación tienen la capacidad de resolver problemas de carácter común, como la falsificación y el del doble gasto, a través de la red de mineros la cual valida todas y cada una de las transacciones realizadas bajo esta moneda electrónica, de esto se deduce que gracias a la complejidad matemática que implica el código bajo el cual trabaja Bitcoin no existe forma de falsificar, hacer fraude o modificar las reglas descritas por cada plataforma o monedero virtual sin contar con la aprobación del proveedor de la criptomoneda. 
Este autor también agregó que, en el código madre se tiene un límite de 21 millones de unidades o Bitcoins, el cual ayuda a la prevención de la inflación, del mismo modo, menciona que, es una alternativa para hacer pagos y enviar dinero por todo el mundo de forma libre, sin horarios, sin bancos, con completo control y con comisiones muy bajas. Más aún, debido al esquema de recompensas y comisiones con el que los mineros reciben un pago por sus servicios, muchas personas han visto en dicha actividad una oportunidad de negocio redituable, lo cual aporta cierto grado de auto-sustentabilidad (Pérez, 2018). Así también señalan, que las ventajas que tiene la implementación del Bitcoin son por un lado que, tendrá un límite de emisión que llegará a 21 millones, lo que la convierte en una moneda que tiende a apreciarse frente a otras, es decir, se trata de una moneda con restricción de emisión lo que se traduce en baja inflación y revalorización de la moneda, por otra parte, es una moneda "anónima", por lo que no la puede controlar un gobierno, ni una entidad, otra ventaja señalada por este autor es que el control de las transacciones es realizado por todos los participantes del ecosistema Bitcoin, es decir, cada operación queda completamente registrada, de tal manera que cualquiera puede ver movimientos, aunque sin poder detectar quién los hace, otra ventaja es que las transacciones se hacen en tiempo real.

\subsection{Desventajas de las transacciones comerciales con Bitcoin}

De acuerdo a Orrala \& Chompol (2017), Lemos (2020), López, Restrepo, \& Ocampo (2020), Forero \& Sanabria (2017), Torres (2015) y Melguizo (2019), las desventajas más visibles son la volatilidad, ya que desde su creación ha tenido grandes subidas de precio, mientras que, lo normal es que con el tiempo el porcentaje de variación se reduzca y tenga una cotización más estable, otra desventaja citada por este autor es la garantía de aceptación, pues aunque cada vez hay más establecimientos que los aceptan todavía son una minoría, quién advirtió que no se existe garantía de que sea una moneda aceptada de forma legal por todos los países, por otra parte, señaló como otra desventaja el hecho de que no cuenta con respaldo de activos físicos o emisores legales, así como también que, el tipo de cambio es impredecible, donde los activos como el Bitcoin no tienen valor intrínseco ni ofrecen un pago final prometido ni dividendos, lo que sería una gran desventaja, puesto que no sirven, en general, como moneda de curso legal o como un medio de intercambio común y oficial. Aunque actualmente el país de Guatemala oficializó en el mes de setiembre del año 2021 el curso legal de la moneda Bitcoin como 
forma de intercambio. No obstante, siguen las dudas, sobre todo, sobre los riesgos que supone su implementación, sobresaliendo la integridad fiscal y financiera ya que, que pueden estar asociadas al lavado de activos y la evasión tributaria.

Estos autores también coinciden en que la volatilidad y la garantía de aceptación de esta moneda aun es desconocida en el mercado financiero, así como el desarrollo en curso de este software, ya que, aún está en fase beta con muchas características incompletas en desarrollo, además actualmente es escasa su implementación en el comercio, al igual que el hecho de que, las criptomonedas sean relativamente nuevas, por lo que aún se encuentran en desarrollo para ofrecer mayor seguridad y poder ser accesible a la mayoría de las personas.

Por su parte, Triviño \& López (2020), Agila (2019), Farías \& Espitia (2018) y Pérez (2018), coinciden en mencionar las siguientes desventajas: Su falta de regulación que amenaza la estabilidad económica y financiera global, debido a su anonimato puede ser usado con fines ilícitos, pues es comúnmente asociado con mercados ilícitos, lo que genera desconfianza, la irreversibilidad de las transacciones y la seguridad y/o garantía de los monederos (e-wallets). Por ende se puede deducir su aspecto legal como principal desventaja, por lo que genera cierta incertidumbre, más aún porque tratándose de sistemas informáticos, es vulnerable a los ataques informáticos, pudiendo ser los usuarios, víctimas de ataques informáticos a sus monederos o plataformas de intercambio.

\subsubsection{Desventajas del Aspecto Técnico del Bitcoin.}

Por otra parte, en lo que respecta a las investigaciones en un aspecto técnico. Estas se han centrado en comprender la dinámica de las redes de Bitcoin, haciendo intentos por vencer el anonimato -área en la cual ya hay avances- y combatir las malas prácticas como lavado de dinero y fraude, toda vez que dichas prácticas han generado un estigma social hacia este sistema, sin embargo, es importante recordar que no son exclusivas del sistema, ya que el dinero en efectivo es igualmente utilizado para fines ilícitos (Pérez, 2018).

Ahora bien, en su análisis FODA, Oña (2019) planteó las siguientes desventajas o debilidades internas: Poca credibilidad. Poca garantía de los recursos y la utilización de los mismos. Poco uso por parte de los usuarios. Mientras que, también agregó las siguientes amenazas externas: La desconfianza de la población, no permite que se desarrolle el sistema. Las personas prefieren usar otros medios de pagos diferentes al efectivo (dinero plástico). Falta de difusión de información. 
Mientras que las desventajas citadas por Izquierdo (2018) fueron: a) Su enorme fluctuación en el valor contra otras monedas. b) No hay garantías de que se convierta en una moneda aceptada por todos. c) La falta de un regulador del ecosistema explica en gran medida la volatilidad, como depende exclusivamente de la oferta y la demanda, se corre el riesgo de que sus variaciones desalienten el uso. d) El anonimato que garantiza el sistema no permite saber quiénes están detrás de las operaciones, de esta manera, un profesional independiente que cobrara sus servicios en Bitcoin, un gobierno no podría detectarlo para cobrarle impuestos, pero tampoco detectaría a un narcotraficante.

\section{CONCLUSIONES Y RECOMENDACIONES.}

Los estudios consultados indican que el Bitcoin está ganando terreno como la moneda digital del futuro, sigue en etapa de prueba tentativa en las masas, lo que da tiempo a seguir observando el comportamiento de los mercados, procesos sociales, políticos, culturales y tecnológicos que pudieran o no viabilizar el uso masivo generalizado como medio de transacción.

Las principales desventajas según las fuentes consultadas que presenta la moneda digital Bitcoin, son: falta de credibilidad, poca garantía de los recursos y la utilización de los mismos, poco uso por parte de los usuarios, fluctuación en el valor contra otras monedas, falta de una base legal normativa y de una base financiera.

Con el análisis documental realizado, se puede concluir que el bitcoin se encuentra en proceso de experimentación, que independientemente de su fiabilidad o no, pregona numerosas ventajas y desventajas que sirven para atraer al público objetivo, lo que, de confirmar, a corto o largo plazo las bondades de este tipo de moneda, se podría predecir el futuro del sistema económico dentro de los entornos virtuales, siendo el bitcoin el de mayor promoción.

No obstante, está claro que las principales desventajas del uso de la moneda virtual que reportan las fuentes consultadas son las siguientes: falta de regulación y sus divergencias, tanto como ventajas y desventajas, escasos estudios sobre experiencias de usuarios. Este último, hace alejada la idea de criticar este sistema innovador de transacción comercial sin tomar en cuenta la perspectiva, ideas y experiencias de los usuarios, que si bien, no se ha demostrado científicamente su factibilidad, es innegable que está acaparando espacios en el mundo financiero y comercial a nivel mundial. 
En cuanto a las principales ventajas en la que coinciden los autores son: su globalidad, no pertenece a ningún estado o gobierno, es ajeno al sistema fiduciario, su valor, las transacciones en tiempo real, cuenta con estándares de seguridad y riesgos, alto grado de confianza cibernético, bajo costos de transacción, facilidad de acceso, libertad de pagos, tasas muy bajas, menores riesgos para los comerciantes, seguridad de las transacciones, irreversibles, confidencialidad de datos personales y privados de los clientes, sin intermediarios y transacción en tiempo récord, el dinero no es embargable, y no tiene costo de almacenamiento.

Dado la discrepancia entre los autores, respecto a la ventaja y desventaja de la utilización del bitcoin en los procesos comerciales, surgieron algunas dudas sobre la investigación respecto la aceptación por parte de los comercios, la experiencia del usuarios con el bitcoin y la capacidad de respuesta del sistema de trasferencia de dicha moneda, al respecto se proponen nuevas líneas de investigación que podrán responder a interrogantes que surgen a partir de las conclusiones alcanzadas: ¿Cuál es la experiencia de los usuarios reales del bitcoin en sus procesos comerciales? ¿Cómo las empresas perciben el impacto del bitcoin en sus finanzas? ¿Evidencian los empresarios y usuarios del bitcoin mayor ventaja en relación a las desventajas del bitcoin como moneda electrónica en los procesos comerciales?

\section{LISTA REFERENCIA}

Agila, R. (2019). El Bitcoin: Crecimiento y desarrollo de la criptomoneda y su inclusión como alternativa de pago y medio de intercambio en la economía ecuatoriana, período: 2014-2018. Guayaquil: Universidad de Guayaquil. Facultad de Ciencias Económicas. Obtenido de http://repositorio.ug.edu.ec/handle/redug/40599

Artigas, L., Lucas Almudi Higueras, I., \& Sánchez Chóliz, J. (2015). ¿ Son las monedas virtuales el dinero del futuro? Consultado de https://zaguan.unizar.es/record/48074

Armijos, V. (2015). Las monedas virtuales en las transacciones comerciales. Un análisis sobre el uso de nuevos sistemas de pagos. Research Gate.

Canales, M. P. (2020). El BITCOIN: Comprendiendo el Mercado. Apuntes de economía y sociedad, 1(1), 9-24. 
Callejo, J. (2020). El uso de criptomonedas en 142 países las convierte en fenómeno global. Consultado https://observatorioblockchain.com/bitcoin/bitcoin-conviertelas-criptomonedas-en-fenomeno-global-142-paises-las-usan/

Compains, F. J. R., García, V. E. S., \& Campo, S. R. (2021). Futuros de Bitcoin: Aspectos a considerar en su valoración ante una posible creación de un mercado europeo. RUE: Revista universitaria europea, (34), 137-160.

Cajamarca Andrade, D. M., \& Curipoma Narváez, D. S. (2020). Análisis socioeconómico en la aplicación del Bitcoin como forma de pago en medianas empresas de Guayaquil" (Bachelor's thesis, Universidad de Guayaquil Facultad de Ciencias Administración).

Carrera-López, J. S., Sánchez-Lunavictoria, J. C., \& Loza-Torres, A. G. (2020). El uso de las criptomonedas como nueva forma de pago en la economía mundial. Revista Científica FIPCAEC (Fomento de la investigación y publicación en Ciencias Administrativas, Económicas y Contables). ISSN: 2588-090X. Polo de Capacitación, Investigación y Publicación (POCAIP), 5(5), 210-223.

Canales Gutiérrez, S. (2018). Bitcoin, la moneda descentralizada de curso voluntario, como equivalente funcional del peso colombiano. Departamento de Derecho.

Cadena Díaz, P. A., \& Rincón León, H. A. (2018). ¿ Qué Son Las Criptomonedas?.

Criollo, M. J., \& Maldonado, M. S. (2021). Probabilidad de la implementación del Bitcoin a través de eCommerce en Ecuador. Guayaquil: Universidad Católica de Santiago de Guayaquil. Obtenido de http://201.159.223.180/handle/3317/16229

Díaz, K. C. C., Franco, F. D. J. M., \& Díaz, G. M. B. REFLEXIONES RESPECTO AL TRATAMIENTO FISCAL DE LAS CRIPTOMONEDAS EN MÉXICO.

Farías, C. A., \& Espitia, M. F. (2018). Aceptación del BITCOIN como medio de pago. Bogotá: UNIVERSIDAD LIBRE FACULTAD DE CIENCIAS ECONÍMICAS, ADMINISTRATIVAS Y CONTABLES INSTITUTO DE POSGRADOS. Obtenido de https://repository.unilibre.edu.co/handle/10901/15929

Forero, M., \& Sanabria, J. (2017). VENTAJAS Y DESVENTAJAS DE LA APLICACIÓN DE LAS CRIPTOMONEDAS “BITCOIN” EN EL ORDENAMIENTO JURIDICO TRIBUTARIO DE COLOMBIA . Santa Marta: UNIVERSIDAD DEL MAGDALENA. 
Gracia, S. G. (2018) Análisis Jurídico, Fiscal y Financiero del Bitcoin A Legal, Fiscal and Financial Analysis of Bitcoin.

Gonzales I. A. P. (2018). Criptomonedas: naturaleza jurídica y riesgos en la regulación de su comercialización. Revista de derecho del mercado de valores, (22), 5

Ibarra Muñoz, P. L. (2019). Bitcoin: Perspectivas de los alumnos de la Facultad de Ciencias Económicas de la Universidad Nacional del Este.

Izquierdo, E. (2018). BITCOIN. Universidad Miguel Hernández de Elche. Obtenido de http://193.147.134.18/bitstream/11000/7411/1/TFG-

Izquierdo\%20Cervera\%2C\%20Enrique.pdf

Juanpere, B. A. (2019). La fiscalidad de bitcoin en España. Crónica Tributaria, 173(4), 735.

J. Pérez de Lama (2018). Diagrama explicando cómo funciona una transacción con Bitcoin. Extraído de https://arquitecturacontable.wordpress.com/2018/02/28/introduccion_bitcoin-yblockchain/

Laise, L. D., \& Manzo-Ugas, G. (2019). Bases para la interpretación y regulación razonable de las criptomonedas: naturaleza, dificultades y desafíos constitucionales. Cuadernos del Cendes, (100), 107-124.

Licona, D. R. E., \& Sosa, F. A. P. (2019). El bitcoin, $\measuredangle$ una burbuja especulativa? Análisis de la estabilidad paramétrica de series de tiempo para el periodo 2009-2018. Eseconomia. Revista de estudios económicos, tecnológicos y sociales del mundo contemporáneo, 14(51), 45-61

Lemos, J. F. (2020). Descripción de las ventajas y riesgos de la inversión del BITCOIN en Colombia entre 2014 y 2019. Santiago de Cali: Universidad Santiago de Cali. Obtenido de https://repository.usc.edu.co/handle/20.500.12421/5099

López, A., Restrepo, J. M., \& Ocampo, M. (2020). La criptomonedas: Ventajas y desventajas para la economía de las personas y las empresas en la ciudad de Medellín. Medellín: Tecnológico de Antioquia Institución Universitaria. Facultad de Ciencias Administrativas y Económicas. Obtenido de https://dspace.tdea.edu.co/handle/tda/561

Lozano, D. A. V., \& Forero, I. G. F. Tributación bitcoin: una comparación entre Colombia y Estados Unidos. 
Melguizo, C. A. (2019). Ventajas y desventajas de las criptomonedas en la economía colombiana. Bogotá: Universidad Militar Nueva Granada. Obtenido de https://repository.unimilitar.edu.co/handle/10654/35279

Montoya Ramírez, J. D. (2020). El bitcoin una innovación financiera (Bachelor's thesis, Escuela de Economía, Administración y Negocios. Facultad de Negocios Internacionales).

Moreno, B., Valencia, N., Soto, F., \& Sánchez, A. (2018). Criptomonedas como alternativa de inversión, riesgos, regulación y posibilidad de monetización en Colombia.

Medina, M. A. C., Zarate, C. V., \& López, L. M. (2020). La necesidad de la regulación contable, legal y fiscal del bitcoin en México, un tema de actualidad. Horizontes de la Contaduría en las Ciencias Sociales, (13).

Muñoz Esteban, M. (2017). La moneda digital: El bitcoin.

Naúmenko, T. V., \& Fakhrutdínova, L. R. (2019). La tecnología Blockchain en América Latina. Iberoamérica, (3), 28-51.

Oña, D. A. (2019). Análisis del impacto causado por la implementación del sistema de dinero electrónico en el Ecuador durante el período 2014-2017. Universidad de Guayaquil. Facultad de Ciencias Económicas. Obtenido de http://repositorio.ug.edu.ec/handle/redug/40915

Orrala, K. d., \& Chompol, L. E. (2017). Análisis de las ventajas del uso del BITCOIN en el Ecuador como una criptomoneda alternativa a las transacciones comerciales. GUAYAQUIL: Universidad De Guayaquil.Facultad De Ciencias Matemáticas Y Físicas.Carrera De Ingeniería En Networking Y Telecomunicaciones. Obtenido de http://repositorio.ug.edu.ec/handle/redug/23667

Pérez, G. (2018). Análisis de la evolución de la criptomoneda bitcoin en el mundo entre el 2010 y el 2018. Bogotá: Fundación Universidad de América. Obtenido de http://52.0.229.99/handle/20.500.11839/6923

Padró Martín, L. (2019). Criptomonedas y tecnología blockchain. La iniciativa "Valladolid Blockchain".

Reyes, M. F. M., \& Joancomartí, J. H. Análisis Y Comparación De Monedas Criptográficas Basadas En La Tecnología Blockchain.

Rodríguez Gómez, J. L. (2020). Bitcoin, un activo de inversión alternativo. 
Reyes Asanza, M. A., \& Rosero Banchon, L. N. (2018). Bitcoin y la nueva era de la economía informática en el Ecuador (Bachelor's thesis).

Rosales, M. S., Maledo, V. G. R., \& García, G. G. BITCOIN: UNA VISIÓN GENERAL.

Sánchez Leonsegui, R. (2018). Análisis teórico-práctico de la moneda virtual Bitcoin y sus implicaciones económicas.

Sánchez, J. Z., Ruiz, S. Y., Osorio, S. A., López, M. C., Arredondo, C. C. R., Carazo, D. F., \& López, J. D. (2019). Aspectos tributarios de las transacciones en criptomonedas: el caso de los bitcoins. Revista Instituto Colombiano de Cerecho Tributario, 43-69.

Sánchez, S. G. O. (2021). Educación financiera basada en el bitcoin y la inclusión en planes de estudio. RIDE Revista Iberoamericana para la Investigación y el Desarrollo Educativo, 11(22).

Saldarriaga, V. P. (2019). Lavado de activos mediante criptomonedas en el Perú: problemas y alternativas. Lex: Revista de la Facultad de Derecho y Ciencia Política de la Universidad Alas Peruanas, 17(24), 161-178.

Torres, E. (2015). Reflexiones respecto a las ventajas y desventajas del uso del Bitcoin. Universidad Piloto de Colombia. Obtenido de http://repository.unipiloto.edu.co/handle/20.500.12277/3750

Triviño, H. Y., \& López, R. E. (2020). Bitcoin: uso y potencialidades en Colombia. Bogotá: Repositorio Institucional UCC. Obtenido de https://repository.ucc.edu.co/handle/20.500.12494/33314

Vázquez-Gutiérrez, M. Á. Inversiones globales en entornos inciertos: El Bitcoin. 